\title{
DESARROLLO DE ELECTRODOS DE BAJO COSTO BASADO EN ÓXIDOS METÁLICOS PARA LA GENERACIÓN DE CLORO
}

\author{
Daniel Bazán Arista ${ }^{a}$, Angélica María Baena Moncada ${ }^{a}$, Adolfo La Rosa-Toro Gómez ${ }^{*}$
}

\begin{abstract}
RESUMEN
Se prepararon electrodos de $\mathrm{SnO}_{2}$ dopados con $\mathrm{Sb}$ soportados en láminas porosas de titanio mediante impregnación, sinterización y descomposición térmica de soluciones precursoras con diferentes porcentajes en masa de $\mathrm{Sn}$ y $\mathrm{Sb}(\mathrm{Sn}: \mathrm{Sb}=9: 1$ y $\mathrm{Sn}: \mathrm{Sb}=7: 3)$. Estos fueron recubiertos por una capa de $\mathrm{B}-\mathrm{PbO}_{2}$ obtenida por el método de electrodeposición anódica a una temperatura de $65{ }^{\circ} \mathrm{C}$ y una densidad de corriente $30 \mathrm{~mA} \mathrm{~cm}{ }^{-2}$, durante 30 minutos. Los electrodos fueron caracterizados por espectroscopía Raman, microscopía electrónica de barrido y voltamperometria cíclica. Los resultados obtenidos mostraron que el recubrimiento con mayor contenido de estaño brinda las condiciones óptimas para la formación de la inter-capa entre el $\mathrm{Ti}$ y el $\mathrm{PbO}_{2}$, como la formación de una película homogénea, bajos sobrepotenciales para la reacción de evolución de cloro y estabilidad a pH neutro. La película de $\mathrm{PbO}_{2}$ electro-sintetizada presentó estructura piramidal y de acuerdo al espectro Raman presenta la fase dominate de Plattnerite $\left[\beta-\mathrm{PbO}_{2}\right]$, desarrolla bajos sobrepotenciales para la reacción de evolución de cloro con un tiempo de vida determinado por cronopotenciometría de 171,75 horas en electrólisis en $\mathrm{NaCl}$ al $6 \%$ empleando una densidad de corriente de 30 $\mathrm{mA} \mathrm{cm}{ }^{-2}$.
\end{abstract}

Palabras clave: dióxido de estaño, dióxido de plomo, reacción de oxidación de cloro, tiempo de vida.

\section{DEVELOPMENT OF ANODIC ELECTRODES BASED ON LOW COST METAL OXIDES FOR THE GENERATION OF FREE CHLORINE}

\begin{abstract}
$\mathrm{SnO}_{2}$ electrodes doped with $\mathrm{Sb}$ were prepared, supported on porous titanium sheets by impregnation, sintering and thermal decomposition of precursor solutions with different mass percentages of $\mathrm{Sn}$ and $\mathrm{Sb}(\mathrm{Sn}: \mathrm{Sb}=9: 1$ and $\mathrm{Sn}: \mathrm{Sb}=7: 3)$. The electrodes were coated with a layer of $\mathrm{B}-\mathrm{PbO}_{2}$ obtained by anodic electrodeposition method at $65^{\circ} \mathrm{C}$ and a current density of $30 \mathrm{~mA} \mathrm{~cm}{ }^{-2}$, for 30 minutes. The electrodes were characterized by Raman spectroscopy,
\end{abstract}

\footnotetext{
a Universidad Nacional de Ingeniería, Av. Túpac Amaru s/n, Rímac, Lima 25, Perú toro@uni.edu.pe
} 
scanning electron microscopy, and cyclic voltammetry. The results obtained showed that the catalyst with the highest content of $\mathrm{Sn}$ provides the optimal conditions for the interlayer formation between $\mathrm{Ti}$ and $\mathrm{PbO}_{2}$, such as the formation of a homogeneous film, low overpotentials for the chlorine evolution reaction and stability at neutral $\mathrm{pH}$. The synthesized $\mathrm{PbO}_{2}$ film presented a pyramidal shape morphology, according to the Raman spectrum; it has the Plattnerite form $\left[\beta-\mathrm{PbO}_{2}\right]$. In addition to low overpotentials for the chlorine evolution reaction, with a life time determined by Cronopotenciometric measurements of 171.75 hours in $\mathrm{NaCl}$ at $6 \%$ and with a current density of $30 \mathrm{~mA} \mathrm{~cm}^{-2}$.

Key words: tin dioxide, lead dioxide, chlorine oxidation reaction, lifetime

\section{INTRODUCCIÓN}

En la actualidad, la producción electroquímica mundial de cloro es un proceso que consume grandes cantidades de energía y emplea electrodos basados en metales nobles, por lo que es necesario optimizar el proceso empleando elcetrodos de bajo costo y de buena eficiencia anódica. Los anodos dimensionalmente estables son electrodos que se emplean para la producción industrial de cloro ${ }^{1}$. Los electrodos de $\mathrm{Ti} / \mathrm{SnO}_{2}-\mathrm{Sb}_{2} \mathrm{O}_{3}$ son uno de los materiales más prometedores para ser empleados en el proceso de oxidación electroquímica, estos materiales presentan altos sobrepotenciales para la reacción de evolución de oxígeno, comportamiento que se encuentra asociado a la generación de radicales hidroxilo; la elevada eficiencia obtenida se le atribuye a la buena conductividad del componente de óxido de antimonio; además de tener bajo costo y baja toxicidad. Sin embargo, presentan la desventaja de tener un tiempo de vida muy corto $^{1-3}$. En este contexto, el material del ánodo juega un papel crítico, porque este deberá ser inocuo, de bajo costo y deberá tener un tiempo de vida útil.

El óxido de plomo existe en dos estados de oxidación, $\mathrm{Pb}^{4+}\left(\mathrm{PbO}_{2}\right)$ y $\mathrm{Pb}^{2+}(\mathrm{PbO})$, toma dos formas cristalinas, tetragonal $\left(\beta-\mathrm{PbO}_{2}, \alpha-\mathrm{PbO}\right)$ y ortorrómbico $\left(\beta-\mathrm{PbO}_{2}, \alpha-\mathrm{PbO}\right)^{4,5}$. El óxido de plomo ha sido usado como material anódico, es de bajo costo, estable y fácil de preparar, además posee altos sobrepotenciales para la reacción de evolución de oxígeno ${ }^{6}$. Muchos investigadores han hecho un gran esfuerzo por aumentar el desempeño de los electrodos de $\mathrm{PbO}_{2}$, como la adición de una capa interna $\left(\mathrm{SnO}_{2}-\mathrm{Sb}\right)$ entre el titanio y el $\mathrm{PbO}_{2}$, o la incorporación de un dopante metálico o no metálico ${ }^{7,8}$. Otros estudios han demostrado que el uso de un sustrato de Ti poroso tiene un papel importante en el desempeño del $\mathrm{PbO}_{2}{ }^{9}$.

En este trabajo se utilizó el método de descomposición térmica como método de síntesis para obtener los óxidos de estaño dopados con antimonio sobre las placas de Ti porosas, donde se probó dos proporciones diferentes de $\mathrm{Sn}: \mathrm{Sb}$ 9:1 y Sn:Sb 7:3, siendo más estable el electrodo con mayor porcentaje de $\mathrm{Sn}$; posteriormente, se electrodepositó una película de $\beta-\mathrm{PbO}_{2}$ sobre la superficie de $\mathrm{SnO}_{2}-\mathrm{Sb}_{2} \mathrm{O}_{3}$. Los óxidos depositados fueron evaluados frente a la reacción de evolución de cloro por voltamperometría cíclica, y el tiempo de vida útil a pH neutro por cronopotenciometría. 


\section{PARTE EXPERIMENTAL}

\section{Preparación de electrodos de $\mathrm{Ti} / \mathrm{SnO}_{2}-\mathrm{Sb}_{2} \mathrm{O}_{3} / \mathrm{PbO}_{2}\left(\mathrm{EA}-\mathrm{Sn}_{\mathrm{x}} \mathrm{Sb}_{\mathrm{y}} / \mathrm{Pb}\right)$}

Como soporte se utilizó titanio poroso (99,99\%, Goodfellow) (tamaño: $40 \mathrm{~mm}$ x $5 \mathrm{~mm} \mathrm{x}$ $0,5 \mathrm{~mm})$. Las láminas de titanio fueron tratadas con ácido oxálico al $10 \%\left(\mathrm{C}_{2} \mathrm{H}_{2} \mathrm{O}_{4 \cdot 2} \mathrm{H}_{2} \mathrm{O}\right.$; 99,9 \%, J.T.BAKER) en ebullición, y se lavó con agua ultrapura (Sistema MILLIPORE Q MILLI PLUS, $18,2 \mathrm{~m} \Omega \mathrm{cm}^{-1}$ a $25^{\circ} \mathrm{C}$ ). La capa interna de $\mathrm{SnO}_{2}-\mathrm{Sb}_{2} \mathrm{O}_{3}$ fue depositada por descomposición térmica sobre el titanio poroso $\mathrm{Ti} / \mathrm{SnO}_{2}-\mathrm{Sb}_{2} \mathrm{O}_{3}$, denotado como TD- $\mathrm{Sn}_{\mathrm{x}} \mathrm{Sb}_{\mathrm{y}}$, partiendo de una solución de $\mathrm{SnCl}_{2} \cdot 2 \mathrm{H}_{2} \mathrm{O}$ (98-101 \%, Fermont), $\mathrm{SbCl}_{3}$ (>99\%, Merck) y $\mathrm{HCl}$ concentrado (36-37 \%, Merck), se utilizó como solvente etanol (89-91\%, Fermont) ${ }^{10,11}$. Los porcentajes nominales de los precursores fueron (en moles) DT-Sn $9 \mathrm{Sb}_{1}$ ( $\mathrm{Sn}: \mathrm{Sb}=9: 1$ ) y DT-Sn ${ }_{6} \mathrm{Sb}_{4}(\mathrm{Sn}: \mathrm{Sb}=6: 4)$. El electrodo poroso de titanio fue impregnado con la solución precursora utilizando un pincel, con posterior secado a $80{ }^{\circ} \mathrm{C}$ por un periodo de 5 min. Luego se realizó un tratamiento térmico a $400{ }^{\circ} \mathrm{C}$ por $10 \mathrm{~min}$ en presencia de oxígeno. El proceso se repitio 30 veces hasta alcanzar una carga másica entre $1.1-1.6 \mathrm{mg} \mathrm{cm}^{-2}$. Al alcanzar la carga másica deseada se realizó un tratamiento final a $500{ }^{\circ} \mathrm{C}$ durante $1 \mathrm{~h}^{10}$. Finalmente se obtuvo como producto una capa intermedia de $\mathrm{SnO}_{2}-\mathrm{Sb}_{2} \mathrm{O}_{3}$.

Una vez obtenidos los electrodos de DT- $\mathrm{Sn}_{\mathrm{x}} \mathrm{Sb}_{\mathrm{y}}$, se procedió a electrodepositar una capa de $\beta-\mathrm{PbO}_{2}$ para obtener electrodos del tipo Ti/ $\mathrm{SnO}_{2}-\mathrm{Sb}_{2} \mathrm{O}_{3} / \mathrm{PbO}_{2}\left(\mathrm{EA}-\mathrm{Sn}_{9} \mathrm{Sb}_{1} / \mathrm{Pb}_{65}\right)$. Para el depósito de la película de $\mathrm{PbO}_{2}$ sobre los electrodos de $\mathrm{DT}-\mathrm{Sn}_{\mathrm{x}} \mathrm{Sb}_{\mathrm{y}}$ se utilizó un área efectiva de $1 \mathrm{~cm}^{2}$, el depósito se hizo en modo galvanostático en una celda electrolítica de un solo compartimento ${ }^{12}$, utilizando como catodo acero inoxidable, con una separación entre electrodos de $1 \mathrm{~cm}$. La solución electrolítica empleada fue $\mathrm{Pb}\left(\mathrm{NO}_{3}\right)_{2} 0,5 \mathrm{~mol} \mathrm{~L}-1$ (>99,0 \%, ACS reagent SIGMA-ALDRICH) y el $\mathrm{pH}$ se ajusto a 1 con $\mathrm{HNO}_{3}(62-63 \% \text {, pro analysis })^{12}$. Se realizó un estudio del proceso de electrodeposición variando las densidades de corriente desde 5 hasta $30 \mathrm{~mA} \mathrm{~cm}^{-2}$, también se estudió el depósito a diferentes velocidades de agitación desde 0 a $450 \mathrm{rpm}$ y dos temperaturas distintas 25 y $65^{\circ} \mathrm{C}^{11}$. Los electrodos obtenidos fueron lavados con agua desionizadas y secados en estufa ${ }^{13}$.

\section{Caracterización fisicoquímica}

Para estudiar la morfología y hacer un análisis elemental se utilizó un microscopio electrónico de barrido (SEM, JEOL, JSM-6390A) equipado con un analizador de dispersión de rayos X (EDX). Las medidas de dispersión Raman fueron obtenidas con un espectrómetro HORIBA Scientific, utilizando un laser de Ar a una longitud de onda de $632 \mathrm{~nm}$.

\section{Caracterización electroquímica}

Los experimentos electroquímicos fueron llevados a cabo en una celda de electroquímica de tres electrodos, usando un electrodo de referencia de $\mathrm{Ag} / \mathrm{AgCl}$ y grafito como electrodo auxiliar. En este estudio el electrodo de trabajo consistió en un electrodo de Ti modificado (DT-Sn $\left.\mathrm{Sb}_{\mathrm{y}}, \mathrm{EA}-\mathrm{Sn}_{9} \mathrm{Sb}_{1} / \mathrm{Pb}_{65}\right)$. Las medidas electroquímicas fueron realizadas con un potenciostato-galvanostato Ivium STAT en electrolito previamente burbujeado con nitrógeno para desplazar el oxígeno disuelto. En este trabajo todos los potenciales son reportados versus el electrodo de referencia $\mathrm{Ag} / \mathrm{AgCl}\left(\mathrm{KCl} 3 \mathrm{~mol} \mathrm{~L}^{-1}\right) . \mathrm{NaNO}_{3} 0.2 \mathrm{~mol} \mathrm{~L}^{-1}$ (99,5 \%, MERCK) 
fue usado como electrolito soporte. Se realizaron voltamperometrías cíclicas y se hicieron adiciones de $\mathrm{NaCl}$ para observar el comportamiento del electrodo frente a la generación de cloro. $\mathrm{NaCl}$ fue adicionado al electrolito soporte para obtener una concentración final de 0 , 1000,5000 y 10000 ppm.

\section{Evaluación de la estabilidad anódica de los electrodos (tiempo de vida)}

La estabilidad anódica frente la evolución de cloro fue evaluada mediante cronopotenciometría en $2000 \mathrm{~mL}$ de $\mathrm{NaCl} 6 \% \mathrm{w} / \mathrm{v}$, las medidas fueron realizadas a $\mathrm{pH} 7$ usando un potenciómetro $\mathrm{S} / \mathrm{M}$ PL-700PV, el área expuesta de los electrodos fue de $1 \mathrm{~cm}^{2}$ y se impuso una corriente constante de $150 \mathrm{~mA} . \mathrm{cm}^{-2}$ para todos los electrodos a $25^{\circ} \mathrm{C}$. El análisis por cronopotenciometría fue utilizado para registrar el incremento de potencial de los electrodos y se utilizó un Datalogging Hantek 365A, con electrodo de referencia de $\mathrm{Ag} / \mathrm{AgCl}\left(\mathrm{KCl} 3 \mathrm{~mol} \mathrm{~L}^{-1}\right)$.

\section{RESULTADOS Y DISCUSIÓN}

\section{Síntesis y caracterización de los electrodos DT- $\mathrm{Sn}_{\mathrm{x}} \mathrm{Sb}_{\mathrm{y}}$}

Una lámina de titanio porosa fue utilizada como sustrato, primero se desengrasó con acetona sumergiéndolo durante $10 \mathrm{~min}$, posteriormente se atacó químicamente con ácido oxálico al $10 \% \mathrm{w} / \mathrm{t}$ durante una hora en ebullición. El tratamiento produjo una superficie rugosa de color gris apropiada para el anclaje de los óxidos. Para obtener los óxidos metálicos se empleó sales precursoras de $\mathrm{SnCl}_{2} \cdot 2 \mathrm{H}_{2} \mathrm{O}$ y $\mathrm{SbCl}_{3}$ en una relación de $\mathrm{Sn} / \mathrm{Sb} 6: 4$ y $\mathrm{Sn} / \mathrm{Sb}$ 9:1 en masa, las cuales fueron disueltas en etanol, $\mathrm{HCl}$ fue adicionado para asegurar la completa disolución de las sales. Las soluciones precursoras fueron impregnadas en el sustrato poroso de titanio mediante pintado, mojando bien la superficie sin dejar excesos, seguido de un secado a $80{ }^{\circ} \mathrm{C}$ durante 5 minutos para eliminar el solvente y posterior tratamiento térmico a $400{ }^{\circ} \mathrm{C}$ entre capa y capa, con un tratamiento final a $500{ }^{\circ} \mathrm{C}$ durante una hora con el propósito de obtener la sinterización de los óxidos. El análisis de EDX mostró que las composiciones en masa de los óxidos obtenidos eran $\mathrm{Sn} / \mathrm{Sb}$ 9:1 para el primer electrodo y $\mathrm{Sn} / \mathrm{Sb}$ 7:3 para el segundo electrodo (tabla 1). En la figuara 1 se puede observar las imágenes SEM de los electrodos sintetizados, para el electrodo de $\mathrm{DT}-\mathrm{Sn}_{9} \mathrm{Sb}_{1}$ (figura $2 \mathrm{~A}$ y B) se observa una película homogénea y de mejor calidad que el obtenido con el electrodo DT- $\mathrm{Sn}_{7} \mathrm{Sb}_{3}$ (figura 1 $\mathrm{C}$ y D), donde se observa huecos y fragmentos grandes dentro de la película. 

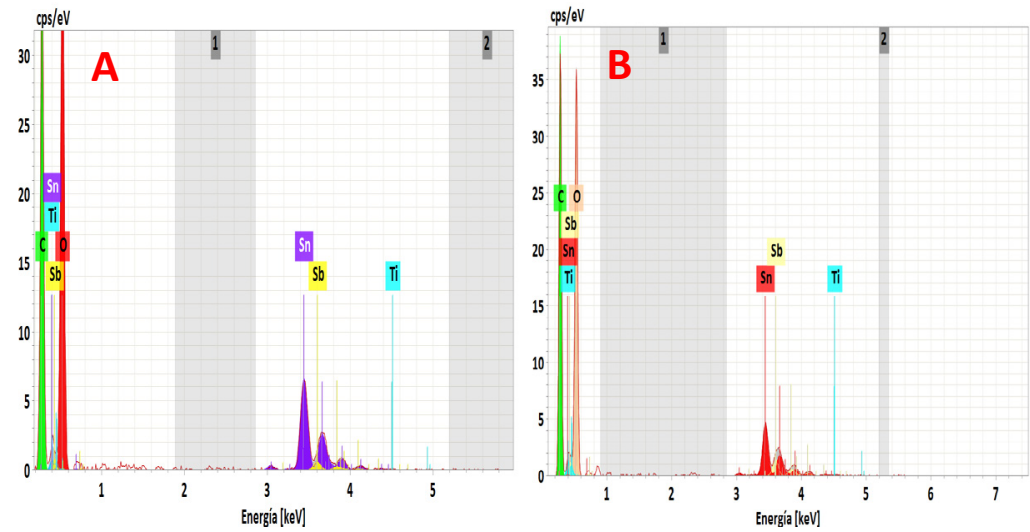

Figura 1. Imagen EDX para los catalizadores DT- $\mathrm{Sn}_{\mathrm{x}} \mathrm{Sb}_{\mathrm{y}}$. A) Catalizador DT- $\mathrm{Sn}_{9} \mathrm{Sb}_{1}, \mathrm{~B}$ ) Catalizador DT- $\mathrm{Sn}_{7} \mathrm{Sb}_{3}$

tabla 1. Porcentaje en masa obtenido por análisis EDX para los electrodos DT- $\mathrm{Sn}_{9} \mathrm{Sb}_{1}$ y DT- $\mathrm{Sn}_{7} \mathrm{Sb}_{3}$

\begin{tabular}{ccc}
\hline Electrodo & DT-Sn ${ }_{9} \mathrm{Sb}_{1}$ & $\mathrm{DT}-\mathrm{Sn}_{7} \mathrm{Sb}_{3}$ \\
\hline Elemento & Porcentaje en masa / \% & Porcentaje en masa $\%$ \\
\hline $\mathrm{Sb}$ & 6,87 & 21,42 \\
\hline $\mathrm{O}$ & 27,12 & 22,76 \\
\hline $\mathrm{Ti}$ & 4,60 & 7,80 \\
\hline $\mathrm{Sn}$ & 61,40 & 48,02 \\
\hline Proporción en masa $\mathrm{Sn}: \mathrm{Sb}$ & $90: 10$ & $69: 31$ \\
\hline Proporción en masa $\mathrm{Sn}: \mathrm{Sb}$ & $90: 10$ & $60: 40$ \\
nominal & &
\end{tabular}




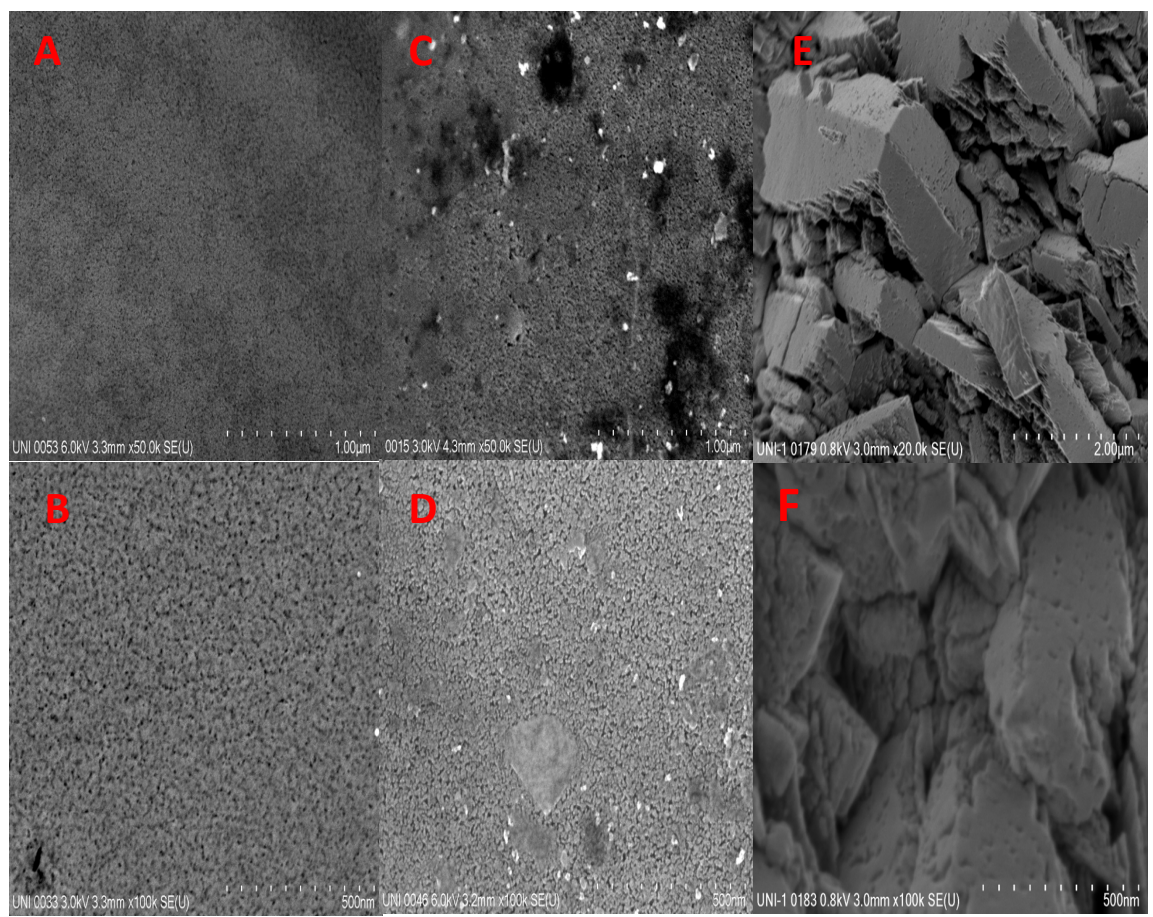

Figura 2. Imagen SEM de los catalizadores sintetizados. A y B corresponden al catalizador DT$\mathrm{Sn}_{9} \mathrm{Sb}_{1}, \mathrm{C}$ y D al catalizador DT-Sn $\mathrm{Sb}_{3}$. E y F al catalizador EA- $\mathrm{Sn}_{10} \mathrm{Sb}_{1} / \mathrm{Pb}_{65}$

Las voltamperometrías cíclicas en $\mathrm{NaNO}_{3} 0.2 \mathrm{~mol} \mathrm{~L}^{-1}$, de ambos electrodos, se muestran en la figura 3, se puede observar que el potencial de inicio para la reacción de evolución de oxígeno (REO) son 1,43 V y 1,56 V para DT-Sn9Sb1 y DT-Sn7Sb3, respectivamente. Para observar la actividad catalítica para la reacción de evolución de $\mathrm{Cl}_{2}$ de acuerdo con:

\section{$2 \mathrm{Cl}^{-} \rightleftarrows \mathrm{Cl}_{2}+2 \mathrm{e}^{-}$}

Se realizaron adiciones de $\mathrm{NaCl}$, se puede apreciar en la figura 2 A) y B) un aumento de corriente, a medida que aumenta la concentración de $\mathrm{NaCl}$, a menores concentraciones de $\mathrm{NaCl}$ el sobrepotencial para la $\mathrm{REO}$ es mayor, indicando una mayor inhibición para la oxidación de oxígeno ${ }^{3}$. También se observa una disminución en el potencial de inicio de la oxidación cuando se agregan $1000 \mathrm{ppm} \mathrm{NaCl}$ de 0,01 y $0,05 \mathrm{~V}$ para DT-Sn $\mathrm{Sb}_{1}$ y DT-Sn $\mathrm{Sb}_{3}$, respectivamente, este comportamiento puede ser atribuido al hecho de que la corriente de oxidación corresponde no solo a la formación de oxígeno, involucrando también a la evolución de cloro, ambos procesos ocurren de forma simultánea y en competencia. En la figura $3 \mathrm{C}$ se puede apreciar un gráfico de la densidad de corriente versus en potencial de inicio de la oxidación, en donde se puede observar que el electrodo DT- $\mathrm{Sn}_{9} \mathrm{Sb}_{1}$, presenta menores sobrepotenciales para la reacción de evolución de cloro y densidades de corriente de pico más altas que el electrodo DT- $\mathrm{Sn}_{7} \mathrm{Sb}_{3}$. 

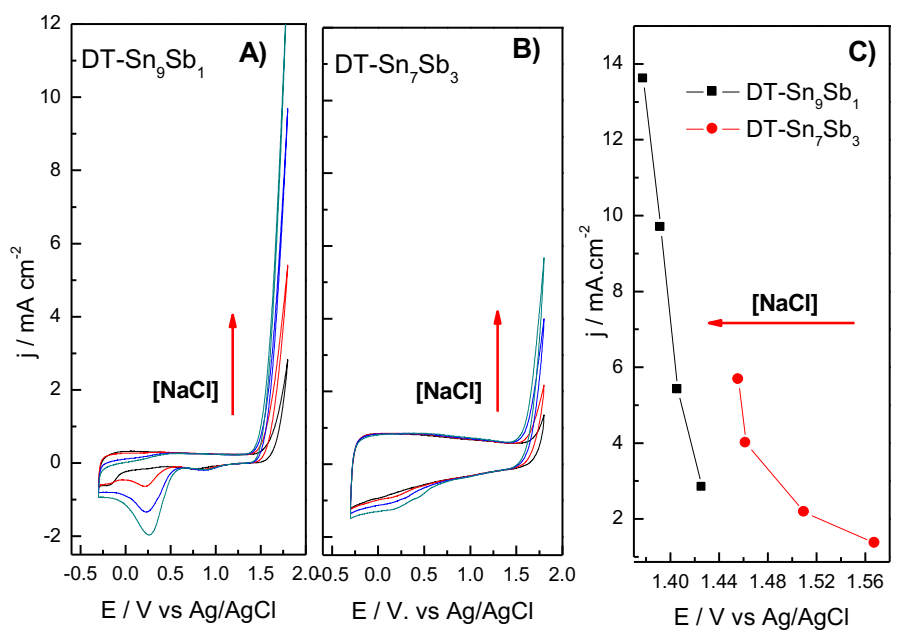

Figura 3. Voltamperometrías cíclicas en $\mathrm{NaNO}_{3} 0,2$ mol L-1 para los electrodos $\mathrm{DT}-\mathrm{Sn}_{\mathrm{x}} \mathrm{Sb}_{\mathrm{y}^{*}}$. Línea negra 0 ppm NaCl, Línea roja 1000 ppm NaCl, Línea azul 5000 ppm NaCl, Línea verde $10000 \mathrm{ppm} \mathrm{NaCl}$. A). DT- $\mathrm{Sn}_{9} \mathrm{Sb}_{1}, \mathrm{~B}$ ) DT-Sn $\mathrm{Sb}_{3}$.C) Grafico de la densidad de corriente versus el inicio de la oxidación, $v: 50 \mathrm{mV} \mathrm{s}^{-1}$.

\section{Síntesis y caracterización del electrodo $\mathbf{E A - S n _ { 9 }} \mathbf{S b}_{1} / \mathbf{P b}_{65^{\circ}}$}

La electrodeposición de $\mathrm{PbO}_{2}$ sobre los electrodos de DT- $\mathrm{Sn}_{x} \mathrm{Sb}_{y}$ se realizó en una celda electrolítica usando dos láminas de acero como cátodo. El ánodo (DT- $\mathrm{Sn}_{\mathrm{x}} \mathrm{Sb}_{\mathrm{y}}$ ) se colocó entre las dos láminas durante el proceso de deposición anódica. De acuerdo a los resultados obtenidos durante la caracterización morfológica y electroquímica de los electrodos DT$\mathrm{Sn}_{9} \mathrm{Sb}_{1}$ y DT- $\mathrm{Sn}_{7} \mathrm{Sb}_{3}$, se observó que el electrodo de DT-Sn $\mathrm{Sb}_{1}$ mostró un mejor cubrimiento de la película de $\mathrm{PbO}_{2}$ depositada, buena adhesión al sustrato y excelente cubrimiento superficial, además de menores sobrepotenciales para la reacción de evolución de cloro. Por estas razones se eligió el electrodo $\mathrm{DT}-\mathrm{Sn}_{9} \mathrm{Sb}_{1}$ para la electrodeposición del $\mathrm{PbO}_{2}$. La formulación de la solución electrolítica de $\mathrm{Pb}^{2+}$ genera una película de $\mathrm{PbO}_{2}$ de buena calidad a $25^{\circ} \mathrm{C}$. La deposición del film de $\mathrm{PbO}_{2}$ inicialmente se realizó a $25^{\circ} \mathrm{C}, 300 \mathrm{rpm}$ y se varió la densidad de corriente con la finalidad de evaluar las condiciones óptimas para obtener una buena cobertura del sustrato, se usaron densidades de 5, 10, 20 y $30 \mathrm{~mA} \mathrm{~cm}^{-2}$. En la figura 4 se observa que la cantidad de masa electrodepositada es proporcional a la corriente aplicada, como consecuencia se obtiene una línea recta. 

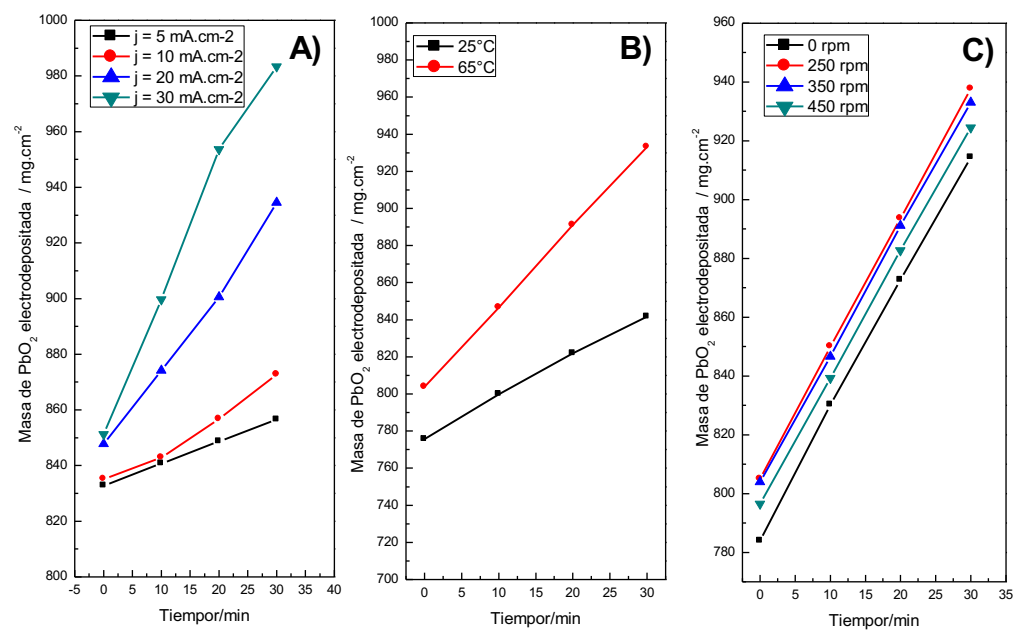

Figura 4. Gráfico de la masa de $\mathrm{PbO}_{2}$ electrodepositada vs. El tiempo de electrodeposición en $0,5 \mathrm{~mol} \mathrm{~L}^{-1} \mathrm{~Pb}\left(\mathrm{NO}_{3}\right)_{2}, \mathrm{pH}$ 1. A). Condiciones de electrolisis: $\mathrm{T}=25^{\circ} \mathrm{C}, \mathrm{t}=120 \mathrm{~min}, 300 \mathrm{rpm}, \mathrm{j}$ $=5,10,20$ y $30 \mathrm{~mA} \mathrm{~cm}^{-2}$. B). Condiciones de electrolisis:: $\mathrm{j}=30 \mathrm{~mA} \mathrm{~cm}^{-2}, \mathrm{t}=30 \mathrm{~min}, \mathrm{v}=300$ $\mathrm{rpm}, \mathrm{T}=25^{\circ} \mathrm{C}$ and $65^{\circ} \mathrm{C}$. C). Condiciones de electrolisis:: $\mathrm{j}=30 \mathrm{~mA} \mathrm{~cm}{ }^{-2}, \mathrm{~T}=65^{\circ} \mathrm{C}, \mathrm{t}=30$ min, v: 0, 250, 300 and $450 \mathrm{rpm}$.

De acuerdo a los test preliminares, la velocidad de masa de $\mathrm{PbO}_{2}$ electrodepositada no depende de la velocidad de agitación de la solución electrolítica (figura 4C), la masa de $\mathrm{PbO}_{2}$ se mantiene casi constante para todas las velocidades probadas. La cantidad de $\mathrm{PbO}_{2}$ depende de la densidad de corriente aplicada, además, la morfología de la película de $\mathrm{PbO}_{2}$ depende de la densidad de corriente, de la concentración de $\mathrm{Pb}$ (II) y la temperatura de la solución electrolítica ${ }^{11}$.

El tiempo de electrolisis no afecta la capa activa de $\mathrm{PbO}_{2}$, y se mantiene la forma típica piramidal reportada por otros autores ${ }^{14}$ pero tiene un pequeño efecto sobre el tipo de cristal, pero no influencia la estructura o el tamaño del cristal. Un buen cubrimiento superficial del sustrato se alcanza después de 10 minutos. Se eligió un periodo de 30 minutos para la electrodeposición del $\mathrm{PbO}_{2}{ }^{11}$. De esta forma se garantiza la cobertura del sustrato y se obtiene un electrodo de $\mathrm{PbO}_{2}$ poroso. Otros autores emplearon tiempos de 60 a 120 minutos de electrolisis conllevando a conseguir un mayor espesor, factor que puede incidir en un mayor tiempo de vida útil ${ }^{13,15}$.

La masa de $\mathrm{PbO}_{2}$ obtenida a $25{ }^{\circ} \mathrm{C}$ fue de $841,51 \mathrm{mg} \mathrm{cm}^{-2}$, mientras que a $65{ }^{\circ} \mathrm{C}$ se obtuvo

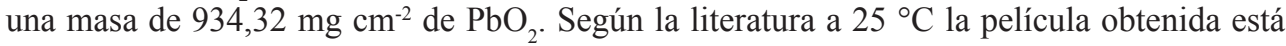
compuesta de partículas casi esféricas poco homogéneas, por encima de los $45^{\circ} \mathrm{C}$ la película obtenida se basa en cristales con forma piramidal. La literatura considera la temperatura de $65^{\circ} \mathrm{C}$ como la temperatura óptima para la electrodeposición ${ }^{11}$. 
En la figura 5 se observa el espectro Raman para los electrodos DT-Sn $\mathrm{Sb}_{\mathrm{y}}$ y el electrodo EA$\mathrm{Sn}_{9} \mathrm{Sb}_{1}-\mathrm{Pb}_{65}$. El espectro del DT-Sn $\mathrm{Sb}_{1}$ muestra tres picos a 439,32, 668,53 y 744,93 $\mathrm{cm}^{-1}$, estos picos están relacionados con la estructura tipo rutilo del $\mathrm{SnO}_{2}$, existe un corrimiento a bajas longitudes de onda debido, probablemente, al reemplazo de iones $\mathrm{Sn}^{2+}$ por iones $\mathrm{Sb}^{2+}$ en la red cristalina ${ }^{16}$. El espectro Raman del catalizador DT- $\mathrm{Sn}_{7} \mathrm{Sb}_{3}$ presenta dos picos adicionales en comparación con el DT-Sn $\mathrm{Sb}_{1}$, un pico a $184 \mathrm{~cm}^{-1}$ y a $467 \mathrm{~cm}^{-1}$, estos picos son debidos al aumento de la concentración de antimonio en la síntesis de este óxido metálico y podría ser asignado a la forma Senarmontite $\left(\mathrm{Sb}_{2} \mathrm{O}_{3} \text { cúbica }\right)^{17}$. En el electrodo EA- $\mathrm{Sn}_{9} \mathrm{Sb}_{1} /$ $\mathrm{Pb}_{65}$ se observan tres picos adiconales a 425,85, 510,84 y $653,87 \mathrm{~cm}^{-1}$ asignados a la forma Plattnerite $[\beta-\mathrm{PbO} 2]^{18}$.

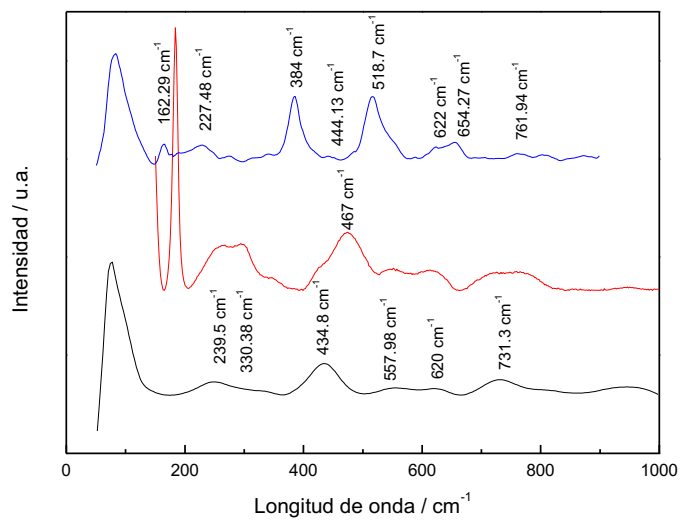

Figura 5. Espectro Raman de los catalizadores DT- $\mathrm{Sn}_{9} \mathrm{Sb}_{1}$, línea negra.DT-Sn $\mathrm{Sb}_{1}$, línea roja y $\mathrm{EA}-\mathrm{Sn}_{7} \mathrm{Sb}_{3} / \mathrm{Pb}_{65}$.

Se realizaron experimentos de voltamperometría cíclica del electrodo $\mathrm{AE}-\mathrm{Sn}_{9} \mathrm{Sb}_{1} / \mathrm{Pb}_{65}$ para observar la estabilidad y el aumento de actividad frente a la producción de cloro en comparación con el electrodo $\mathrm{TD}-\mathrm{Sn}_{9} \mathrm{Sb}_{1}$. El estudio se realizó utilizando $\mathrm{NaNO}_{3}$ como electrolito soporte y con adiciones de diferentes cantidades de $\mathrm{NaCl}$. En la figura 6 se observa la respuesta electroquímica para el electrodo $\mathrm{EA}-\mathrm{Sn}_{9} \mathrm{Sb}_{1} / \mathrm{Pb}$, se produce un corrimiento a potenciales negativos, y la corriente de pico se incrementa cuando se aumenta la concentración de $\mathrm{NaCl}$. Sin embargo, el potencial de inicio para la oxidación se mantiene igual que el obtenido con el electrodo $\mathrm{TD}-\mathrm{Sn}_{9} \mathrm{Sb}_{1}$ como se observa en la figura 3. Se puede apreciar un incremento de corriente para la evolución de cloro en el electrodo EA- $\mathrm{Sn}_{9} \mathrm{Sb}_{1} / \mathrm{Pb}_{65}$ de 16,51 $\mu \mathrm{A}$, en comparación con el valor obtenido en electrodo de TD-Sn $\mathrm{Sb}_{1}(13,54 \mu \mathrm{A})$. 


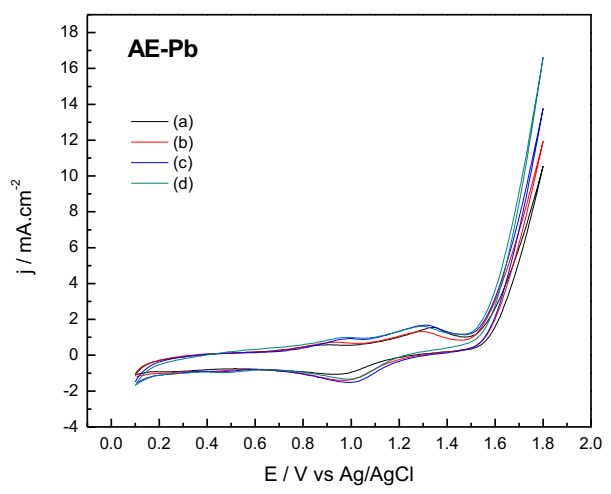

Figura 6. Voltamperometría cíclica en $\mathrm{NaNO}_{3} 0,2 \mathrm{~mol} \mathrm{~L}^{-1}$ del electrodo EA- $\mathrm{Sn}_{9} \mathrm{Sb}_{1} / \mathrm{Pb}_{65}$. (a) 0 ppm NaCl. (b) 1000 ppm NaCl. (c) 5000 ppm NaCl. (d) 10000 ppm NaCl. v: 50 mV s${ }^{-1}$.

\section{Estabilidad electroquímica}

Es importante conocer la estabilidad de los electrodos sintetizados para la evolución de cloro a $\mathrm{pH}$ 7. La estabilidad electroquímica fue evaluada en $\mathrm{NaCl} 6 \%, \mathrm{pH} 7$; a una corriente constante de $150 \mathrm{~mA} \mathrm{~cm}{ }^{-2}$, los experimentos de tiempo de vida acelerada se realizaron por cronopotenciometría. En la figura 7 se puede observar la cronopotenciometría de los electrodos sintetizados, la desactivación se alcanza después de 1 y 0,25 horas para DT$\mathrm{Sn}_{9} \mathrm{Sb}_{1}$ y DT-Sn $\mathrm{Sb}_{3}$, respectivamente. Estos electrodos no son adecuados para trabajar por periodos prolongados de tiempo, dado que no son estables en estas condiciones de operación, indicando que no es posible utilizarlos para la reacción de evolución de cloro. Se eligió trabajar con el electrodo DT- $\mathrm{Sn}_{9} \mathrm{Sb}_{1}$ como soporte para la electrodeposición de la película de $\mathrm{PbO}_{2}$, debido a que es más estable que el DT- $\mathrm{Sn}_{7} \mathrm{Sb}_{3}$ a $\mathrm{pH}=7$. El electrodo EA-Sn $\mathrm{Sn}_{9} \mathrm{Sb}_{1} / \mathrm{Pb}_{65}$ muestra un potencial constante a $\mathrm{pH} 7$ durante 171,75 horas, después de este periodo hay un incremento significativo de voltaje desde 5,15 a 9,42 V, indicando la desactivación del electrodo. Resultados similares se han obtenido con electrodos de Ti/Sb- $\mathrm{SnO} \mathrm{P}_{2} / \mathrm{Pb}_{3} \mathrm{O}_{4}$ con un tiempo de vida de 180 horas en $\mathrm{NaCl} 0,5 \mathrm{~mol} \mathrm{~L}^{-1}{ }^{19}$. Con base en estos resultados es posible la aplicación del electrodo de $\mathrm{EA}-\mathrm{Sn}_{9} \mathrm{Sb}_{1} / \mathrm{Pb}_{65}$, para la obtención de cloro en condiciones de $\mathrm{pH}$ neutro. 


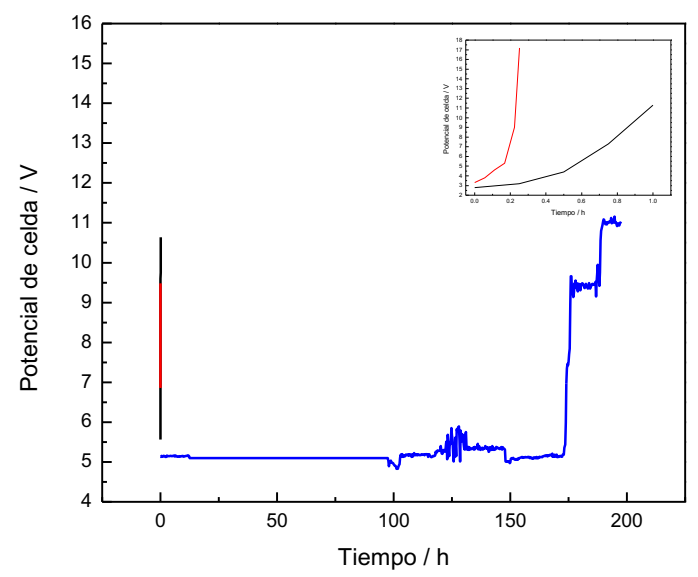

Figura 7. Tiempo de vida en $6 \% \mathrm{NaCl}$ de los electrodos $\mathrm{DT}-\mathrm{Sn}_{9} \mathrm{Sb}_{1}$ línea negra, DT$\mathrm{Sn}_{7} \mathrm{Sb}_{3}$ línea roja y EA-Sn $\mathrm{Sb}_{1} / \mathrm{Pb}_{65}$ línea azul, a corriente constante de $500 \mathrm{mAcm}{ }^{-2}$. Inserto: Ampliación de los electrodos DT- $\mathrm{Sn}_{9} \mathrm{Sb}_{1}$ línea negra, DT-Sn $\mathrm{Sb}_{3}$ línea roja.

\section{CONCLUSIONES}

En este trabajo de investigación se evaluaron dos proporciones de $\mathrm{Sn}$ :Sb para determinar la relación adecuada para la formación de la película que servirá como intercapa entre el titanio y el óxido de plomo. Los resultados mostraron que el electrodo de DT- $\mathrm{Sn}_{9} \mathrm{Sb}_{1}$ presentó las mejores características: Formación de una película homogénea, bajos sobrepotenciales para la reacción de evolución de cloro, y mayores densidades de corriente de pico en comparación al electrodo DT- $\mathrm{Sn}_{7} \mathrm{Sb}_{3}$, permitiendo su empleo como sustrato para la electrodeposición del $\mathrm{PbO}_{2}$. La electrodeposición de $\mathrm{PbO}_{2}$ se realizó a $30 \mathrm{~mA} \mathrm{~cm}{ }^{-2}, 65^{\circ} \mathrm{C}, 300 \mathrm{rpm}$, se obtuvo una película con una forma típica piramidal, por espectroscopía Raman se determinó la fase de Plattnerite $\left[\beta-\mathrm{PbO}_{2}\right]$. Los tres electrodos sintetizados son activos para la reacción de evolución de cloro, sin embargo, los electrodos de DT- $\mathrm{Sn}_{\mathrm{x}} \mathrm{Sb}_{\mathrm{y}}$ no presentaron tiempos de vida adecuados para su utilización, mientras que, el electrodo $\mathrm{EA}-\mathrm{Sn}_{9} \mathrm{Sb}_{1} / \mathrm{Pb}_{65}$ muestra un potencial constante a pH 7 durante 171,75 horas, siendo una buena propuesta como catalizador para reacción de evolución de cloro.

\section{AGRADECIMIENTO}

Los autores agradecen el soporte económico brindado por Cienciactiva bajo el convenio FONDECYT 209-2015 y el apoyo del Dr. Clemente Luyo en los análisis de caracterización SEM y EDX. 


\section{REFERENCIAS BIBLIOGRÁFICAS}

1. Zeradjanin AR, La Mantia F, Masa J, Schuhmann W. Utilization of the catalyst layer of dimensionally stable anodes-Interplay of morphology and active surface area. Electrochim Acta. 2012;82:408-14.

2. Comninellis C. Electrocatalysis in the electrochemical conversion/combustion of organic pollutants for waste water treatment. Electrochim Acta. 1994;39(11-12):1857-62.

3. Shao D, Li X, Xu H, Yan W. An improved stable $\mathrm{Ti} / \mathrm{Sb}-\mathrm{SnO}_{2}$ electrode with high performance in electrochemical oxidation processes. RSC Adv. 2014;4(41):21230-7.

4. D'Antonio P, Santoro A. Powder neutron diffraction study of chemically prepared $\beta$-lead dioxide. Acta Crystallogr Sect B. 1980;36(10):2394-7.

5. Filatov S, Bendeliani N, Albert B, Kopf J, Dyuzeva T, Lityagina L. High-pressure synthesis of $\alpha-\mathrm{PbO} 2$ and its crystal structure at 293, 203, and $113 \mathrm{~K}$ from single crystal diffraction data. Solid state Sci. 2005;7(11):1363-8.

6. Zhou M, Dai Q, Lei L, Ma C, Wang D. Long life modified lead dioxide anode for organic wastewater treatment: electrochemical characteristics and degradation mechanism. Environ Sci Technol. 2005;39(1):363-70.

7. Ding H, Feng Y, Liu J. Preparation and properties of $\mathrm{Ti} / \mathrm{SnO} 2-\mathrm{Sb}_{2} \mathrm{O}_{5}$ electrodes by electrodeposition. Mater Lett. 2007;61(27):4920-3.

8. Ye Z-G, Meng H-M, Sun D-B. Electrochemical impedance spectroscopic (EIS) investigation of the oxygen evolution reaction mechanism of $\mathrm{Ti} / \mathrm{IrO}_{2}{ }^{+} \mathrm{MnO}_{2}$ electrodes in $0.5 \mathrm{~m} \mathrm{H}_{2} \mathrm{SO}_{4}$ solution. J Electroanal Chem. 2008;621(1):49-54.

9. Braga NA, Cairo CAA, Almeida EC, Baldan MR, Ferreiraa NG. From micro to nanocrystalline transition in the diamond formation on porous pure titanium. Diam Relat Mater. 2008;17(11):1891-6.

10. Qiao Q, Wang L, Shi J, Jin J, Li Y. Properties of Fluoride-Doped $\beta-\mathrm{PbO}_{2}$ Electrodes and their Electrocatalytic Activities in Degradation of Acid Orange II. Int J Electrochem Sci. 2015;10:10639-50.

11. Zhao W, Xing J, Chen D, Jin D, Shen J. Electrochemical degradation of Musk ketone in aqueous solutions using a novel porous $\mathrm{Ti} / \mathrm{SnO}_{2}-\mathrm{Sb}_{2} \mathrm{O}_{3} / \mathrm{PbO}_{2}$ electrodes. J Electroanal Chem. 2016;775:179-88.

12. Zhang W, Kong H, Lin H, Lu H, Huang W, Yin J, et al. Fabrication, characterization and electrocatalytic application of a lead dioxide electrode with porous titanium substrate. $\mathrm{J}$ Alloys Compd. 2015;650:705-11.

13. $\mathrm{Li} \mathrm{X}, \mathrm{Xu} \mathrm{H}$, Yan W. Fabrication and characterization of $\mathrm{PbO}_{2}$ electrode modified with polyvinylidene fluoride (PVDF). Appl Surf Sci. 2016;389:278-86.

14. Hao X, Quansheng Y, Dan S, Honghui Y, Jidong L, Jiangtao F, et al. Fabrication and characterization of $\mathrm{PbO}_{2}$ electrode modified with $\left[\mathrm{Fe}(\mathrm{CN})_{6}\right]^{3-}$ and its application on electrochemical degradation of alkali lignin. J Hazard Mater. 2015;286:509-16.

15. Shen Y, Li F, Li S, Liu D, Fan L, Zhang Y. Electrochemically enhanced photocatalytic degradation of organic pollutant on $\beta-\mathrm{PbO}_{2}-\mathrm{TNT} / \mathrm{Ti} / \mathrm{TNT}$ bifunctional electrode. Int $\mathrm{J}$ Electrochem Sci. 2012;7:8702-12. 
16. Müller V, Rasp M, Štefanić G, Ba J, Günther S, Rathousky J, et al. Highly Conducting Nanosized Monodispersed Antimony-Doped Tin Oxide Particles Synthesized via Nonaqueous Sol- Gel Procedure. Chem Mater. 2009;21(21):5229-36.

17. Cody CA, DiCarlo L, Darlington RK. Vibrational and thermal study of antimony oxides. Inorg Chem. 1979;18(6):1572-6.

18. Burgio L, Clark RJH, Firth S. Raman spectroscopy as a means for the identification of plattnerite $\left(\mathrm{PbO}_{2}\right)$, of lead pigments and of their degradation products. Analyst. 2001;126(2):222-7.

19. Shao D, Yan W, Cao L, Li X, Xu H. High-performance Ti/Sb- $\mathrm{SnO}_{2} / \mathrm{Pb}_{3} \mathrm{O}_{4}$ electrodes for chlorine evolution: Preparation and characteristics. J Hazard Mater. 2014;267:238-44. 